\title{
Fault Tolerance Automotive Air-Ratio Control Using Extreme Learning Machine Model Predictive Controller
}

\author{
Pak Kin Wong, ${ }^{1}$ Hang Cheong Wong, ${ }^{1}$ Chi Man Vong, ${ }^{2}$ Tong Meng Iong, \\ Ka In Wong, ${ }^{1}$ and Xianghui Gao ${ }^{1}$ \\ ${ }^{1}$ Department of Electromechanical Engineering, University of Macau, Macau \\ ${ }^{2}$ Department of Computer and Information Science, University of Macau, Macau \\ Correspondence should be addressed to Pak Kin Wong; fstpkw@umac.mo
}

Received 7 August 2014; Revised 27 September 2014; Accepted 28 September 2014

Academic Editor: Jiuwen Cao

Copyright (C) 2015 Pak Kin Wong et al. This is an open access article distributed under the Creative Commons Attribution License, which permits unrestricted use, distribution, and reproduction in any medium, provided the original work is properly cited.

Effective air-ratio control is desirable to maintain the best engine performance. However, traditional air-ratio control assumes the lambda sensor located at the tail pipe works properly and relies strongly on the air-ratio feedback signal measured by the lambda sensor. When the sensor is warming up during cold start or under failure, the traditional air-ratio control no longer works. To address this issue, this paper utilizes an advanced modelling technique, kernel extreme learning machine (ELM), to build a backup air-ratio model. With the prediction from the model, a limited air-ratio control performance can be maintained even when the lambda sensor does not work. Such strategy is realized as fault tolerance control. In order to verify the effectiveness of the proposed fault tolerance air-ratio control strategy, a model predictive control scheme is constructed based on the kernel ELM backup airratio model and implemented on a real engine. Experimental results show that the proposed controller can regulate the air-ratio to specific target values within a satisfactory tolerance under external disturbance and the absence of air-ratio feedback signal from the lambda sensor. This implies that the proposed fault tolerance air-ratio control is a promising scheme to maintain air-ratio control performance when the lambda sensor is under failure or warming up.

\section{Introduction}

Vehicular emissions are the major source of gaseous pollutants that contribute to the harmful and negative effects on environment and human health. It has been reported in [1-4] that the increasing amount of vehicular emissions has led to hundred thousands of mortalities and billions of economic loss every year. To reduce the amount of toxic elements in vehicular emissions, three-way catalytic converter is currently the most effective after-treatment device. This device reduces the unburned hydrocarbons and carbon monoxide by oxidization and nitrogen oxides by reduction [5]. The conversion efficiency of the catalytic converter, however, depends highly on the air-ratio (also known as lambda). When the airratio is at the stoichiometric value (i.e., air-ratio $=1.0$ ), the conversion efficiency can reach as high as $98 \%$, but derivation of only $1 \%$ from stoichiometry can already result in $50 \%$ degradation on the converter. Therefore, for environmental purpose, the air-ratio is usually regulated to 1 . Meanwhile, as an important engine parameter, the air-ratio should also be controlled to different values for other situations [6]. For instance, if emissions are not concerned, the air-ratio can be regulated to around 0.95 to achieve the best engine power performance, whilst it is 1.05 to achieve the best brake-specific fuel consumption. Consequently, an effective air-ratio control system is necessary for engine system to maintain its best performance under various operating conditions.

Over the past decades, car manufacturers and researchers have developed many air-ratio control strategies [7-12]. Examples include the sliding mode control $[7,8]$, proportional-integral-derivative (PID) control [9], and neuralnetwork-based model predictive control (MPC) [10-12]. Sliding mode control requires a very accurate mathematical definition of the engine model, but in general it is impossible to derive an exact engine dynamics model due to its highly nonlinear nature [13]. In most sliding mode air-ratio control studies, many assumptions have been made in the model derivation, and many coefficients are difficult to determine 
for a real engine, so this strategy may not be suitable for practical use. Although PID control is the most widely used approach in practice, the calibration and tuning of the control parameters are very time-consuming and engine dependent. The tuned PID controller cannot deal with steady disturbance or any change in the engine conditions either. Thus, among these researches, the most appropriate and promising technique for air-ratio control is the neural-network-based $\mathrm{MPC}$, due to its robustness to multivariable, time-varying, and delay systems like modern engine systems [12]. It is wellknown that a reliable prediction model is the core component of the MPC, but the engine models developed in $[10,11]$ were only surrogate models. That is, the models were trained from the data generated by empirical equations rather than a real engine. Therefore, similar to the deficiency of sliding mode control approach, the neural-network prediction models in $[10,11]$ derived from data generated by empirical equations cannot effectively reflect the actual performance of real engines. In the most recent study of MPC aircontrol strategy [12], the prediction model for the controller was constructed based on experimental data rather than numerical data. Experimental results in [12] showed that the controller performance is superior to those of $[10,11]$ in real application. Nevertheless, one major concern for the control strategy in [12] is that the prediction model must rely on the real-time air-ratio signal measured from the lambda sensor located at the exhaust pipe of the engine. When the sensor is under failure or warming up during cold start, the controller becomes ineffective, resulting in poor control performance.

In fact, for most of the current available air-ratio control approaches, the air-ratio measurement must be acquired as the feedback to the controllers. Hence, the problem of lambda sensor failure must be addressed. Although on-board diagnostics for the lambda sensor has been a requirement for more than two decades [14] and any fault of the lambda sensor must be reflected through the "check engine" light on the instrument panel, the driver may not be aware of such fault and may not be willing to replace the lambda sensor when the car can still be driven without significant defect. In that case, the emissions and fuel consumption of that car are already significantly deteriorated. Therefore, maintaining a satisfactory air-ratio control performance when the lambda sensor is under failure or warming up during cold start is of great significance. This paper proposes to build a supplementary air-ratio model to compensate the lambda sensor, in which the measured air-ratio signal is not required as the model input.

From the open literature $[15,16]$, it is possible to predict the air-ratio without using the previous air-ratio signal. For instance, Gassenfeit and Powell [15] compared two algorithms that can predict the air-ratio from either the cylinder pressure time history patterns or the ratio of the cylinder pressure before and after combustion. Another example is the method described by Asik et al. [16], in which the airratio can be roughly estimated from induced crankshaft speed fluctuations. However, the quantities used in these algorithms, say, the in-cylinder pressure and delicate crankshaft speed fluctuation, are usually unavailable in normal vehicle engines because expensive sensors are required. Moreover, as mentioned, empirical equations may not be suitable for real applications. Thus, by following the framework in [12], this study attempts to construct the air-ratio model from experimental data. Extreme learning machine (ELM) [17] is currently a popular and effective algorithm for training model from sample data. Many recent studies [18, 19] already showed that ELM is superior to other famous methods, such as neural-networks, least squares support vector machines, and relevance vector machine, in terms of generalization performance and computational load. ELM has been employed for various practical applications too [20, 21]. Thus, ELM is selected in this study to develop the supplementary air-ratio model.

Among so many variants of ELM, kernel-based ELM is adopted in this study to build the model. It is because, in kernel ELM, the random feature mapping is replaced with a kernel function, so randomness does not occur and the chance of result variations could be reduced [22]. In fact, the model built in [12] was based on an online variant of ELM, whose backbone is simply a basic ELM (i.e., the model is still an ELM with random feature mapping but can be updated when online data is provided). The reason why offline version of ELM is used in this paper instead of the online one is that the real-time data of air-ratio will not be available when the lambda sensor is malfunctioning. As both the air-ratio model from [12] and the proposed air-ratio model are made from the same basis (kernel ELM), a fair comparison can be made to evaluate their performances.

In order to verify the effectiveness of the ELM supplementary air-ratio model, this paper also proposes a nonlinear MPC algorithm for air-ratio control, which utilizes a switch to toggle between the lambda sensor signals and the ELM supplementary air-ratio model predictions. When the lambda sensor works well, the air-ratio measurement will be used; when the lambda sensor is under failure, the backup air-ratio model will be used. The MPC under such strategy is called fault tolerance controller (FTC), which is a novel nontrivial application of ELM. Based on the multiplestep-ahead air-ratio predictions, a control signal is obtained to regulate the air-ratio to trace the desired values. The proposed FTC is also compared with the typical air-ratio control techniques, including online sequential extreme learning machine model predictive controller (OEMPC) [12], diagonal recurrent neural-network model predictive controller (DNMPC) [10], and traditional open-loop air-ratio control system, to evaluate its performance. The concept of kernelbased ELM is provided in Section 2. The construction and evaluation of the ELM supplementary air-ratio model are given in Section 3. The detail of the FTC design is presented in Section 4. Experimental implementation and evaluation of the proposed FTC and OEMPC are provided in Section 5.

\section{Kernel-Based Extreme Learning Machine}

Kernel-based ELM is a learning scheme for single-hiddenlayer feedforward network, with the use of kernel [17]. Considering a set of $N$ training samples $\mathscr{D}=\left\{\left(\mathbf{x}_{i}, t_{i}\right), i=\right.$ $1, \ldots, N\}$, with each $\mathbf{x}_{i}$ being a dimensional input vector 
and $t_{i}$ as the target scalar output, a single-hidden-layer feedforward network with $L$ hidden nodes can be written as

$$
\sum_{k=1}^{L} \beta_{k} h_{k}\left(\mathbf{x}_{i}\right)=\mathbf{h}\left(\mathbf{x}_{i}\right) \boldsymbol{\beta}=t_{i}, \quad i=1, \ldots, N,
$$

where $\mathbf{h}\left(\mathbf{x}_{i}\right)=\left[h_{1}\left(\mathbf{x}_{i}\right), \ldots, h_{L}\left(\mathbf{x}_{i}\right)\right]$ is the feature mapping output with respect to $\mathbf{x}_{i}$ and $\boldsymbol{\beta}=\left[\beta_{1}, \ldots, \beta_{L}\right]^{T}$ is the output weight vector. In kernel-based ELM, this weight vector is determined by minimizing both the norm of the weight vector and the training error. The corresponding optimization problem is

$$
\begin{array}{ll}
\text { Minimize: } & \frac{1}{2}\|\boldsymbol{\beta}\|^{2}+C \frac{1}{2} \sum_{i=1}^{N}\left\|\theta_{i}\right\|^{2} \\
\text { Subject to: } & \theta_{i}=t_{i}-\mathbf{h}\left(\mathbf{x}_{i}\right) \boldsymbol{\beta}, \quad i=1, \ldots, N,
\end{array}
$$

where $C$ is a user-specified penalty term for regularization purpose.

Then, based on the Karush-Kuhn-Tucker theorem, optimizing (2) is equal to solving the following dual optimization problem:

$$
\begin{aligned}
\mathscr{L}_{\mathrm{ELM}}= & \frac{1}{2}\|\boldsymbol{\beta}\|^{2}+C \frac{1}{2} \sum_{i=1}^{N}\left\|\theta_{i}\right\|^{2} \\
& -\sum_{i=1}^{N} \alpha_{i}\left(\mathbf{h}\left(\mathbf{x}_{i}\right) \boldsymbol{\beta}-t_{i}+\theta_{i}\right),
\end{aligned}
$$

where $\alpha_{i}$ is the Lagrange multiplier.

By taking derivatives on (3), the following conditions are obtained:

$$
\begin{aligned}
& \frac{\partial \mathscr{L}_{\mathrm{ELM}}}{\partial \boldsymbol{\beta}}=0 \longrightarrow \boldsymbol{\beta}=\sum_{i=1}^{N} \alpha_{i} \mathbf{h}\left(\mathbf{x}_{i}\right)^{T} \longrightarrow \boldsymbol{\beta}=\mathbf{H}^{T} \boldsymbol{\alpha}, \\
& \frac{\partial \mathscr{L}_{\mathrm{ELM}}}{\partial \theta_{i}}=0 \longrightarrow \alpha_{i}=C \theta_{i}, \quad i=1, \ldots, N, \\
& \frac{\partial \mathscr{L}_{\mathrm{ELM}}}{\partial \alpha_{i}}=0 \longrightarrow \mathbf{h}\left(\mathbf{x}_{i}\right) \boldsymbol{\beta}-t_{i}+\theta_{i}=0, \quad i=1, \ldots, N,
\end{aligned}
$$

where

$$
\boldsymbol{\alpha}=\left[\alpha_{1}, \ldots, \alpha_{N}\right]^{T}, \quad \mathbf{H}=\left[\begin{array}{ccc}
h_{1}\left(\mathbf{x}_{1}\right) & \cdots & h_{L}\left(\mathbf{x}_{1}\right) \\
\vdots & \ddots & \vdots \\
h_{1}\left(\mathbf{x}_{N}\right) & \cdots & h_{L}\left(\mathbf{x}_{N}\right)
\end{array}\right]_{N \times L}
$$

By combining the conditions in (4) and eliminating the Lagrange multipliers $\alpha_{i}$, the optimal weight vector could be calculated as

$$
\boldsymbol{\beta}=\mathbf{H}^{T}\left(\frac{\mathbf{I}}{C}+\mathbf{H H}^{T}\right)^{-1} \mathbf{T},
$$

where $\mathbf{T}=\left[t_{1}, \ldots, t_{N}\right]^{T}$ and $\mathbf{I}$ is the identity matrix.
With (6), the output function of the network for an unknown input $\mathbf{X}$ becomes

$$
f(\mathbf{X})=\mathbf{h}(\mathbf{X}) \mathbf{H}^{T}\left(\frac{\mathbf{I}}{C}+\mathbf{H H}^{T}\right)^{-1} \mathbf{T} .
$$

Finally, by defining a kernel matrix satisfying Mercer's conditions as

$$
\begin{aligned}
\boldsymbol{\Omega}_{\mathrm{ELM}} & =\mathbf{H} \mathbf{H}^{T}: \Omega_{\mathrm{ELM} a, b}=h\left(\mathbf{x}_{a}\right) \cdot h\left(\mathbf{x}_{b}\right) \\
& =K\left(\mathbf{x}_{a}, \mathbf{x}_{b}\right),
\end{aligned}
$$

(7) becomes

$$
f(\mathbf{X})=\left[\begin{array}{c}
K\left(\mathbf{X}, \mathbf{x}_{1}\right) \\
\vdots \\
K\left(\mathbf{X}, \mathbf{x}_{N}\right)
\end{array}\right]^{T}\left(\frac{\mathbf{I}}{C}+\mathbf{\Omega}_{\mathrm{ELM}}\right)^{-1} \mathbf{T} .
$$

In this study, the function $f(\mathbf{X})$ is the supplementary airratio model.

\section{Supplementary Air-Ratio Model}

3.1. Model Construction. The objective of the ELM supplementary air-ratio model is to predict the future air-ratio $y_{p}$ when the lambda sensor is under failure or during cold start. Previous air-ratio measurement must not be used as the inputs to the model. Three engine parameters related closely to the air-ratio performance were carefully selected as the model inputs: fuel injection time (FI), engine speed (ES), and throttle position (TP). The order of the system dynamics was chosen to be 2 (i.e., second-order system with 2 past time steps), which gives the minimum prediction error [12]. The structure of the supplementary air-ratio model is shown in Figure 1.

To build the supplementary air-ratio model, experimental engine data was used rather than empirical equation data. A Honda DC5 Type-R test car with K20A i-VTEC engine was employed to perform the experiment. A MoTeC M800 programmable electronic control unit (ECU) with factory calibration data was used as a base controller to control the engine. The car was run over a dyno test, and totally 5800 data samples were acquired using a wide-band lambda sensor subject to random throttle positions. The first 3000 data samples were used as the training dataset, $\mathscr{D}$, to build the supplementary air-ratio model. The last 2800 data samples were used as the test dataset, $\mathscr{D}_{t}$, to evaluate the generalization of the built supplementary air-ratio model. After training, the output function of the ELM supplementary model for an unseen case can be written in the following form:

$$
y_{p}=\left[\begin{array}{c}
K\left(\mathbf{X}, \mathbf{x}_{1}\right) \\
\vdots \\
K\left(\mathbf{X}, \mathbf{x}_{N}\right)
\end{array}\right]^{T}\left(\frac{\mathbf{I}}{C}+\mathbf{\Omega}_{\mathrm{ELM}}\right)^{-1} \mathbf{T}
$$

where $\mathbf{x}, \mathbf{T} \in \mathscr{D}$ are the training data, $y_{p}$ is the prediction output vector, $\mathbf{X}$ is the unseen input vector, $K\left(\mathbf{x}_{a}, \mathbf{x}_{b}\right)=$ $\exp \left(-\left\|\mathbf{x}_{a}-\mathbf{x}_{b}\right\|^{2} / 2 \sigma^{2}\right)$ is the selected radial basis function 


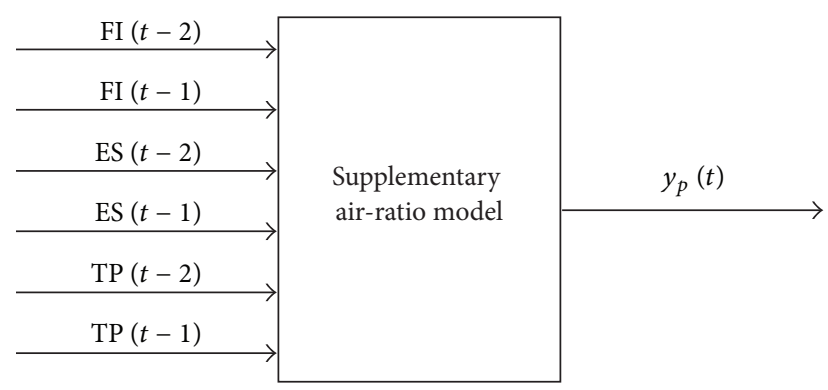

FIGURE 1: Structure of the ELM supplementary air-ratio model.

TABLE 1: Accuracy of different air-ratio models.

\begin{tabular}{lc}
\hline Air-ratio model & LMAE \\
\hline Supplementary air-ratio model & 1.8559 \\
Air-ratio model obtained from [12] & 2.2424 \\
\hline
\end{tabular}

kernel, $\mathbf{I}$ is the identity matrix, and $C$ is the user-specified penalty term. The hyperparameters of $\sigma^{2}$ and $C$ in the kernel-based ELM were tuned by using the hybrid inference introduced in [18], which combined leave-one-out cross validation and Bayesian inference. The details can be found in [18].

3.2. Evaluation of Engine Air-Ratio Model. To evaluate the proposed ELM supplementary air-ratio model, its prediction result was compared with the air-ratio model obtained from [12], which is a time-series model with the air-ratio histories as the input. The performance of the models is evaluated in terms of prediction accuracy for unseen case from $\mathscr{D}_{\mathfrak{t}}$. The prediction results of the two air-ratio models over $\mathscr{D}_{\mathbf{t}}$ are shown in Figure 2.

From Figure 2, it can be seen that both models achieve comparative performance. To further evaluate the prediction accuracy, the logarithmic mean absolute error (LMAE) for the prediction of each model is determined using the following equation:

$$
\text { LMAE }=-\log \left[\frac{1}{T} \sum_{k=1}^{T}\left|y_{k}-y_{p}\right|\right],
$$

where $y_{p}$ is the model prediction value corresponding to $\mathbf{X}_{k}$, $y_{k}$ is the actual experimental value corresponding to $\mathbf{X}_{k}$, and $T$ is total number of predictions from $\mathscr{D}_{t}$, which is $2800 \mathrm{in}$ this case study. The larger the LMAE is, the higher the model accuracy is. The evaluation results are provided in Table 1.

The prediction results from Table 1 show that the prediction accuracy of the proposed ELM supplementary air-ratio model is lower than that of the air-ratio model obtained from [12]. It has to be noted that the dynamics of previous airratio measurement (i.e., $\left.y_{p}(t-1), y_{p}(t-2)\right)$ are not included in the construction of the supplementary air-ratio model. It is reasonable that the prediction accuracy is not as good as the one using these previous air-ratio histories. However, the accuracy is still acceptable, so ELM was confidently selected to implement the FTC in this study.

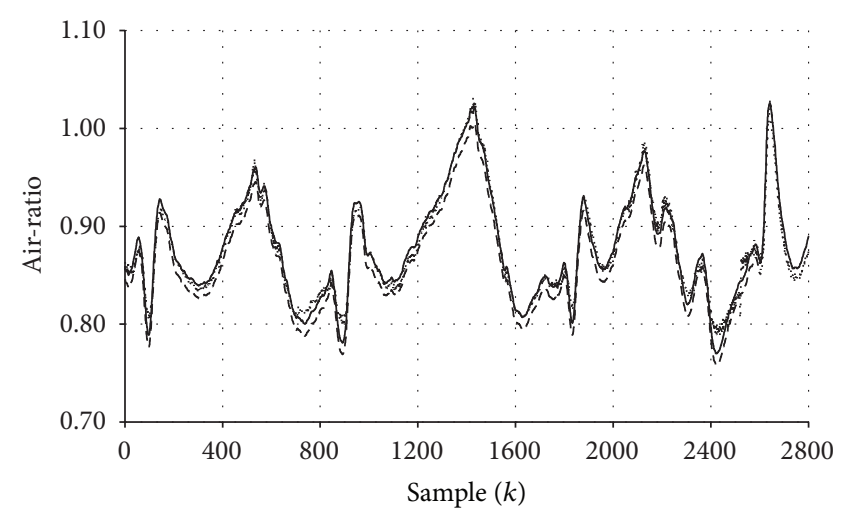

$$
\begin{aligned}
& \text { _ Actual air-ratio } \\
& \text { - - Supplementary model } \\
& \ldots . . \text { Model from (Wong et al., 2014) }
\end{aligned}
$$

FIGURE 2: Comparison between predicted air-ratios and the corresponding actual air-ratios.

\section{MPC Based Fault Tolerance Controller (FTC)}

The proposed FTC can be viewed as an improved version of the OEMPC from [12]. Its aim is to maintain the engine airratio control performance when the lambda sensor is under failure. The design of the proposed FTC is shown in Figure 3.

The FTC consists of two air-ratio models, including the one from [12] and the supplementary air-ratio model from Section 3, a switch toggling between these two models, and an optimizer based on Brent's method [12, 23]. The engine response over a specified time horizon is predicted by either the air-ratio model from [12] or the supplementary air-ratio model, depending on the condition of the lambda sensor. As far as the lambda sensor works well, the engine response is predicted by the air-ratio model from [12]. Normally, the lambda value for a combustible mixture is in the range of 0.4 to 3 [24]. When the lambda measured is out of this range for a firing engine, it means that the lambda sensor is under failure or during cold start. In this situation, the engine response is predicted by the supplementary air-ratio model. The predictions are used by the optimizer to determine the tentative fuel injection time $u^{\prime}$ that minimizes the following performance criterion over the specified time horizon, and then the optimal fuel injection time signal $u$ is sent to the engine:

$$
\begin{aligned}
\min J\left(u^{\prime}\right)= & \sum_{j=N_{1}}^{N_{2}}\left(y_{r}(t+j)-y_{p}(t+j)\right)^{2} \\
& +\rho \sum_{j=1}^{N_{u}}\left(u^{\prime}(t+j-1)-u^{\prime}(t+j-2)\right)^{2},
\end{aligned}
$$

where $N_{1}$ and $N_{2}$ define the prediction horizon, $t$ and $N_{u}$ are the time step and the control horizon, respectively, $y_{r}(t+j)$ is the target air-ratio at the time step $t+j, y_{p}$ is the predicted air-ratio by either of the two air-ratio models at the time step $t+j$, and $\rho$ is a user-defined weight which penalizes excessive 


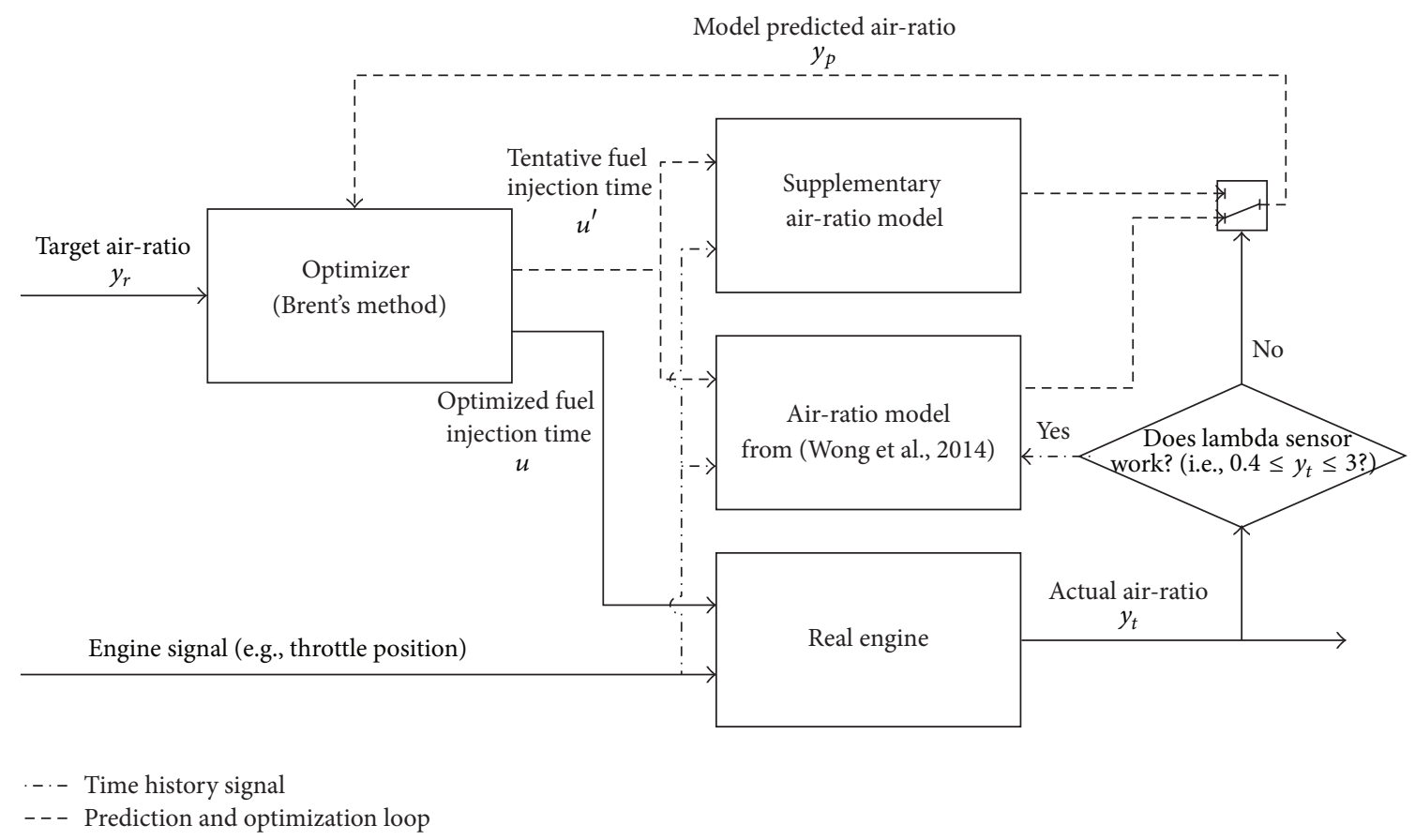

FIgURE 3: Structure of FTC for engine air-ratio control.

movement of the control signal (i.e., the fuel injection time). The variables $u^{\prime}(t+j-1)$ and $u^{\prime}(t+j-2)$ in the second part of (12) are the tentative fuel injection time at the time steps $t+j-1$ and $t+j-2$, respectively. The second part of (12) can ensure the stability of the controller output [12]. Moreover, the stability of MPC strategy has also been proved in $[25,26]$, so the stability of the proposed control scheme could be guaranteed. In short, the proposed control scheme in Figure 3 can provide both regular and fault tolerance control of engine air-ratio.

4.1. Single Dimension Optimization Approach. The original optimization problem involved in this paper is multidimensional and constrained with the tentative control signals, $u^{\prime}(t), u^{\prime}(t+1), \ldots, u^{\prime}\left(t+N_{u}-1\right)$, over the control horizon $N_{u}$ which can minimize the objective function $J\left(u^{\prime}\right)$ of (12). Then the predicted air-ratios, $y_{p}\left(t+N_{1}\right), y_{p}\left(t+N_{1}+\right.$ $1), \ldots, y_{p}\left(t+N_{2}\right)$, can trace the target air-ratios, $y_{r}(t+$ $\left.N_{1}\right), y_{r}\left(t+N_{1}+1\right), \ldots, y_{r}\left(t+N_{2}\right)$, by using the optimized fuel injection time-series. Each fuel injection time is normally bounded within the range from $2 \mathrm{~ms}$ to $15 \mathrm{~ms}$. However, the multidimensional optimization always requires heavy computation, especially when constraints exist. Real-time control applications often put emphasis on computational speed. The research of [10] showed that the one-dimensional approach is efficient for real-time air-ratio control and the overall tracking error is similar to that using multidimensional optimization approach. Therefore, the optimization problem to be solved is reduced to one dimension. In this paper, the control signal $u$ is assumed to remain constant over the control horizon. Therefore, the tentative control signal in the objective function is also constant over the control horizon; that is, $u^{\prime}(t)=u^{\prime}(t+1), \ldots,=u^{\prime}\left(t+N_{u}-1\right)$. In this way, only one parameter $u^{\prime}(t)$ is needed to be determined, and the final fuel injection time at each time step $u$ is set to be the optimal value of $u^{\prime}(t)$

4.2. Brent's Method. There are many optimization techniques available for MPC and each technique has its pros and cons. A well-known technique-Brent's method-was selected as the MPC optimizer in this study for illustrative purpose. Brent's method is a robust and efficient optimization method. It combines the typical parabolic interpolation and goldensection search. The objective function in each iteration is approximated by interpolating a parabola through three existing points. The minimum point of the parabola is taken as a guess for the minimum point if certain criteria are met. Otherwise, golden-section search is carried out. The advantage of this method is that the high convergence rate of parabolic interpolation can be maintained without losing the robustness of golden-section search [23]. The general working principle of Brent's method is shown in Figure 4. The detail optimization procedure of Brent's method was presented in [23] and is not presented herein. There are three parameters of Brent's method, including the initial interval of the input variable, $[a, b]$ (i.e., the limit of the fuel injection time), the tolerance, tol, and the maximum iteration for stopping the optimization procedure. The three variables were set to be $[2,15], 0.05$, and 50 , respectively, because the fuel injection time varies within 2 to $15 \mathrm{~ms}$ from $0 \%$ to $100 \%$ throttle.

\section{Implementation and Evaluation of FTC}

5.1. Experimental Setup. To verify its effectiveness, the proposed FTC was implemented and tested on the same test 


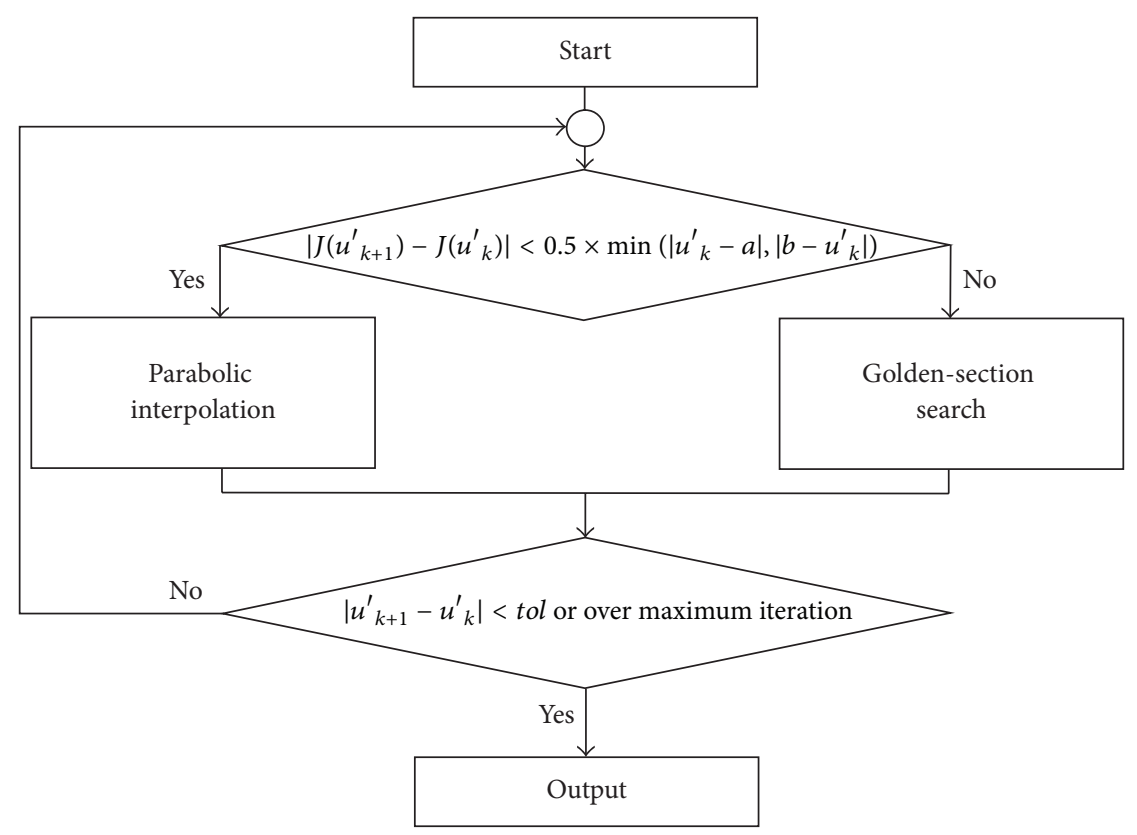

FIGURE 4: General working principle of Brent's method.

car used for sample data collection in Section 3 (Honda DC5 Type-R with K20A i-VTEC engine and MoTeC M800 programmable ECU). The algorithm of FTC was first implemented using MATLAB. A National Instrument (NI) CompactDAQ chassis DAQ-9178 was then employed for signal processing between the MATLAB program and the MoTeC ECU, via NI LabVIEW program. In other words, NI DAQ9178 serves as an interface between the MATLAB program and the MoTeC ECU. Apart from fuel injector control, the MoTeC ECU also contains many basic control maps, such as ignition map and valve timing map, to maintain the engine operation. The experimental setup and the signal flow between the test car and FTC are shown in Figure 5.

5.2. Pilot Test-Tracking Ability. To evaluate the tracking performance of the proposed FTC, a pilot test was carried out. The test was done under the condition that the lambda sensor is already malfunctioning (i.e., the supplementary air-ratio model in the FTC is used). The test cycle for the pilot test is shown in Figure 6, where the throttle position gradually changes from $15 \%$ to $75 \%$ throttle (the throttle position increases by $15 \%$ every $5 \mathrm{~s}$ ). Such test cycle is designed by referring to [10] which almost covers the whole operating condition. In this test, the air-ratio is required to track the target air-ratios from the stoichiometric value (1.00) for minimum emissions to a value for the best brake-specific fuel consumption (1.05) and then to a value for maximum engine power (0.95) as the throttle position is gradually changed from $15 \%$ to $75 \%$ throttle. Such tracking of air-ratio changes is essential for automobiles to satisfy the emission, fuel consumption, and power requirements under different operating conditions [6].
After choosing the sampling time to be $0.01 \mathrm{~s}$, the tracking ability of the FTC was examined. By trial-and-error, the parameters of the optimizer were chosen as $N_{1}=1, N_{2}=8$, $\rho=0.75$, and $N_{u}=5$. With the test cycle shown in Figure 6 and the parameters chosen, the air-ratio control result and the corresponding fuel injection time of the FTC are shown in Figure 7. It can be seen from Figure 7 that the FTC can regulate the air-ratio to follow the target air-ratios with acceptable deviation, even when the lambda sensor is under failure.

To further verify the result, three tests were done. The first one was conducted under the condition that the lambda sensor did not work, and traditional air-ratio controller (i.e., factory lookup table) was employed. The other two were carried out under the condition that the lambda sensor could work well, and a diagonal recurrent neural-network model predictive controller (DNMPC), which is a recent air-ratio control algorithm applicable when the lambda sensor works normally [10], was employed along with the proposed FTC for comparison purpose. The air-ratio control results and the corresponding fuel injection time of the three tests are shown in Figures 8, 9, and 10, respectively.

When comparing Figure 8 to Figure 7, it is obvious that the proposed FTC outperforms traditional air-ratio control system when the lambda sensor does not work. Furthermore, by comparing Figures 9 and 10, it can be seen that the proposed FTC can perform slightly better than the DNMPC when the lambda sensor works properly. Finally, comparing Figure 10 to Figure 7, it can be learnt that the proposed FTC can achieve better performance when the lambda sensor is under normal condition. This is not surprising because when the lambda sensor works well, the air-ratio model from [12], which has better prediction capability, is utilized, resulting in 

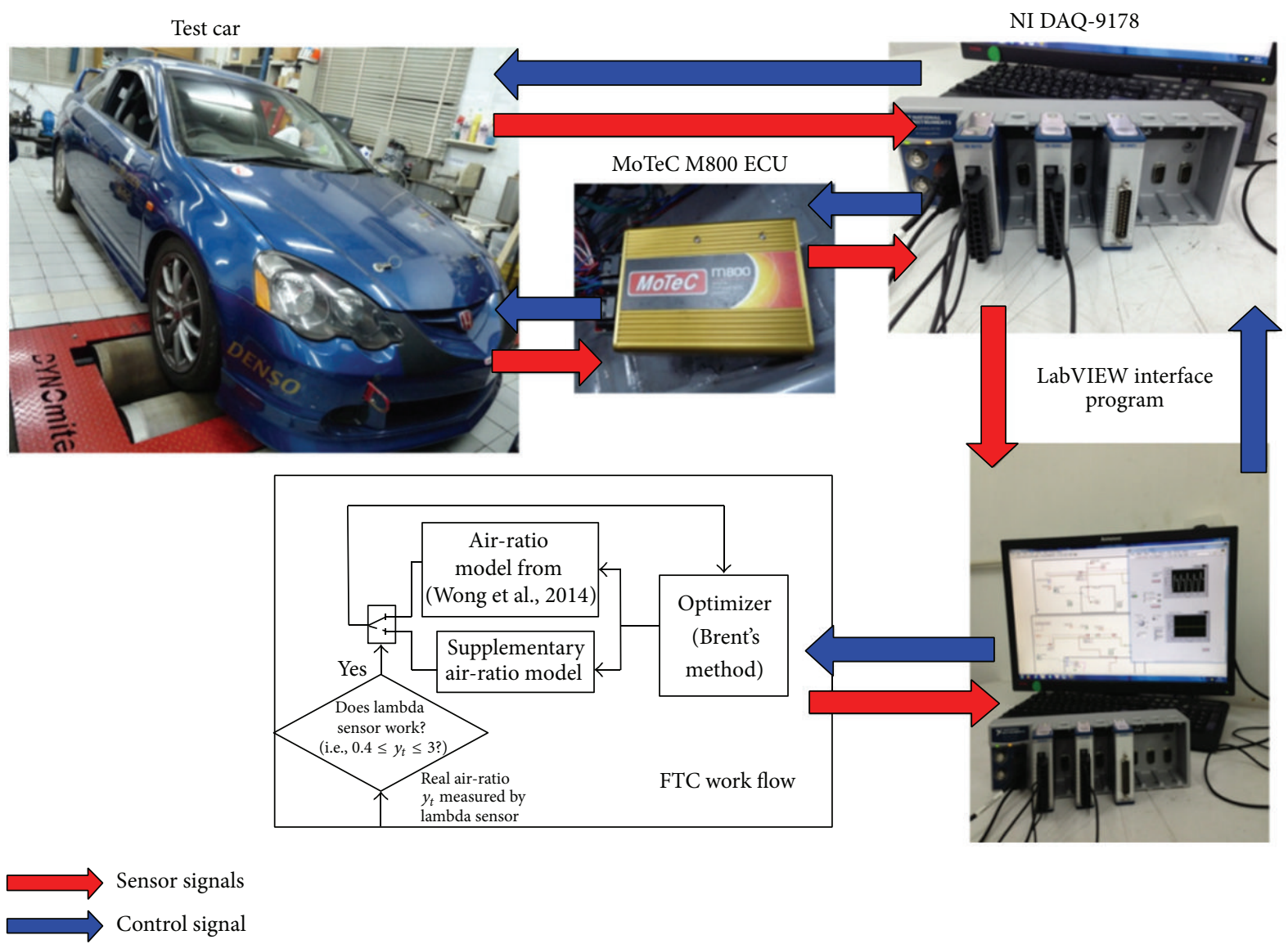

FIGURE 5: Experimental setup and signal flow between test car and FTC.

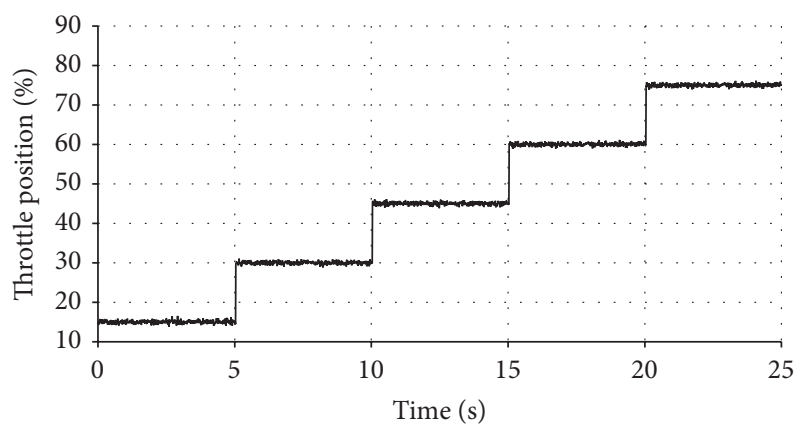

Figure 6: Test cycle: throttle position versus time.

better control performance. For comparison purpose, LMAE is again chosen as the tracking index (TI) to evaluate the tracking ability of the controllers, defined by

$$
\mathrm{TI}=-\log \left[\frac{1}{T_{s}} \sum_{k=1}^{T_{s}}\left|y_{t}-y_{r}(t)\right|\right],
$$

where $t$ is time step, $T_{s}$ is the total number of time step in the test, $y_{t}$ is the actual air-ratio at each time step, and $y_{r}(t)$ is the corresponding target air-ratio at each time step. The target air-ratio varies according to the aforesaid practical operating
TABLE 2: Performance of different controllers for the pilot test.

\begin{tabular}{lccc}
\hline Controller & Lambda sensor & TI & Maximum overshoot \\
\hline FTC & Under failure & 2.1022 & 0.1188 \\
Traditional system & Under failure & 1.6244 & 0.1235 \\
DNMPC & Normal & 2.2896 & 0.1267 \\
FTC & Normal & 2.3309 & 0.1074 \\
\hline
\end{tabular}

conditions. The corresponding results under different lambda sensor conditions are shown in Table 2.

The results in Table 2 show that, even when the lambda sensor is under failure, the control performance of the FTC can still maintain about $90 \%$ of that under normal lambda sensor condition. Table 2 also shows that the performance of the proposed FTC under sensor failure is similar to that of DNMPC. The reason may be that the accuracy of the ELM supplementary model is similar to that of the timeseries diagonal neural-network model in [10]. Moreover, as compared to traditional air-ratio control system (i.e., factory lookup table), the proposed FTC can improve the air-ratio control performance at about $30 \%$ when the lambda sensor is under failure, indicating that the overall air-ratio control performance of the FTC is satisfactory.

Air-ratio model prediction accuracy is one of the most important factors affecting the control performance of 


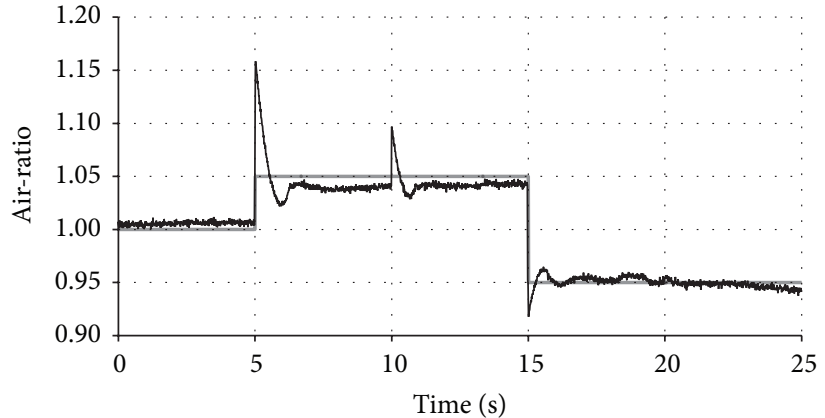

— Target air-ratio

— Actual air-ratio

(a)

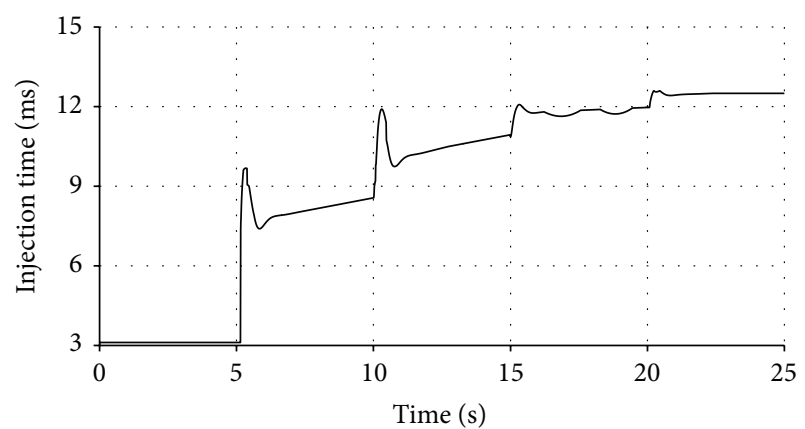

(b)

FIgURE 7: (a) Air-ratio control results and (b) corresponding fuel injection time of FTC under lambda sensor failure.

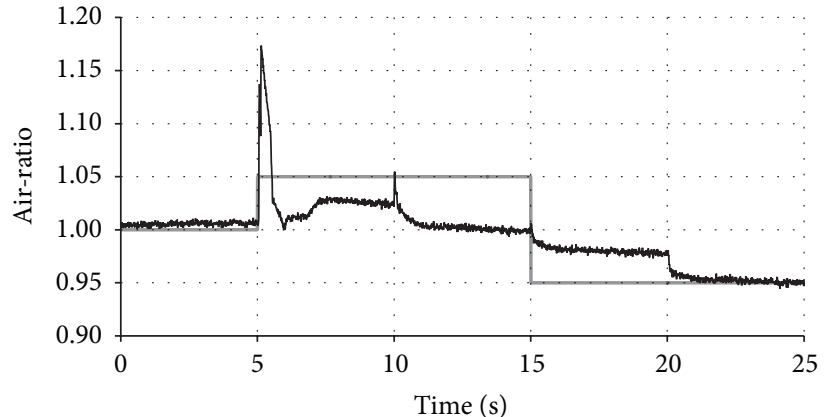

— Target air-ratio

- Actual air-ratio

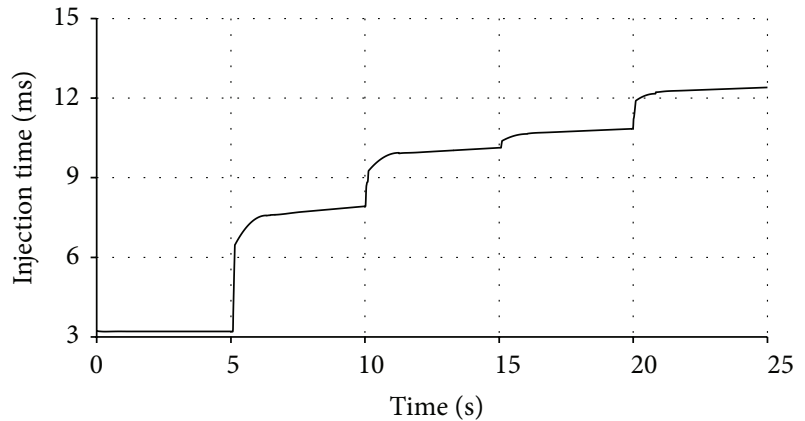

(b)

Figure 8: (a) Air-ratio control results and (b) corresponding fuel injection time of traditional air-ratio control system under lambda sensor failure (i.e., using factory lookup table only).

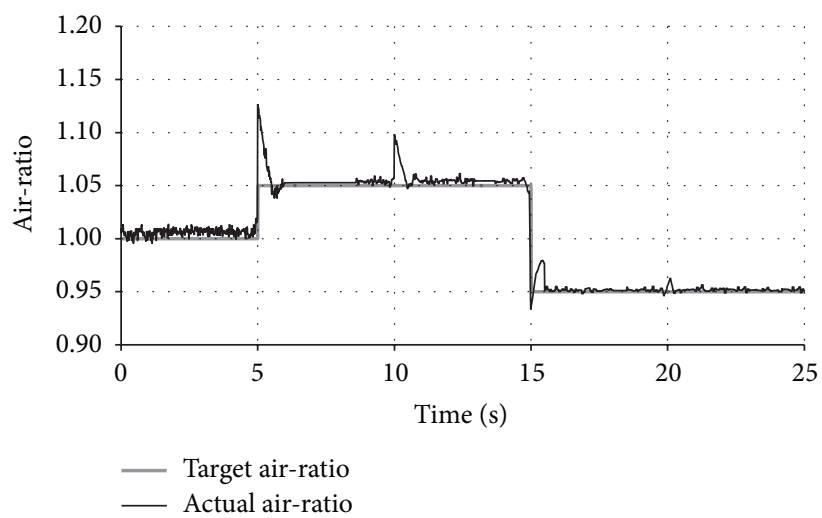

(a)

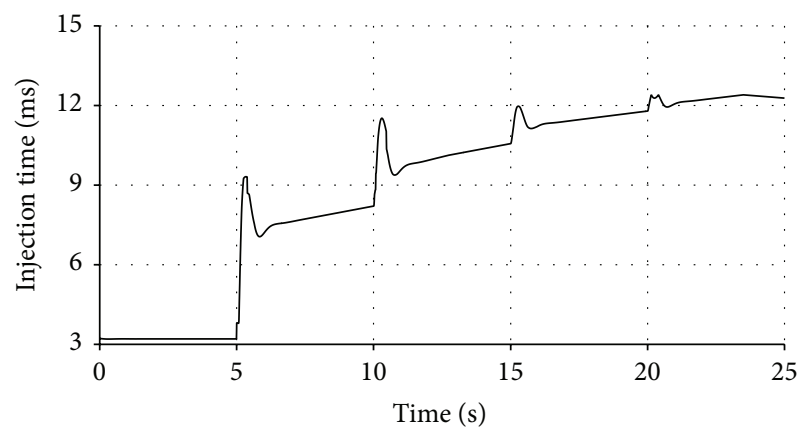

(b)

FIGURE 9: (a) Air-ratio control results and (b) corresponding fuel injection time of DNMPC under normal lambda sensor condition.

the model predictive controllers. Therefore, as the air-ratio measurement cannot be provided for model prediction when the lambda sensor is under failure, degrading of the air-ratio control performance is unavoidable. Thankfully, with the proposed ELM supplementary air-ratio model, the control performance can still be maintained at an acceptable range. This implies that the proposed model and control strategy is feasible. 


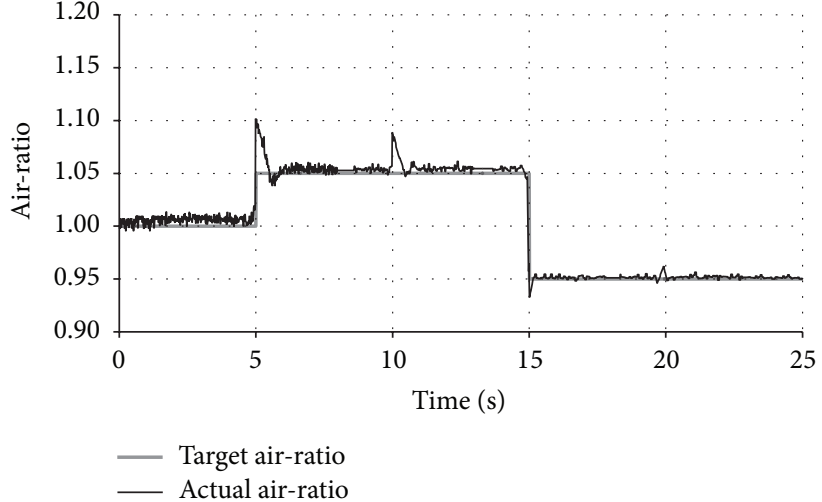

(a)

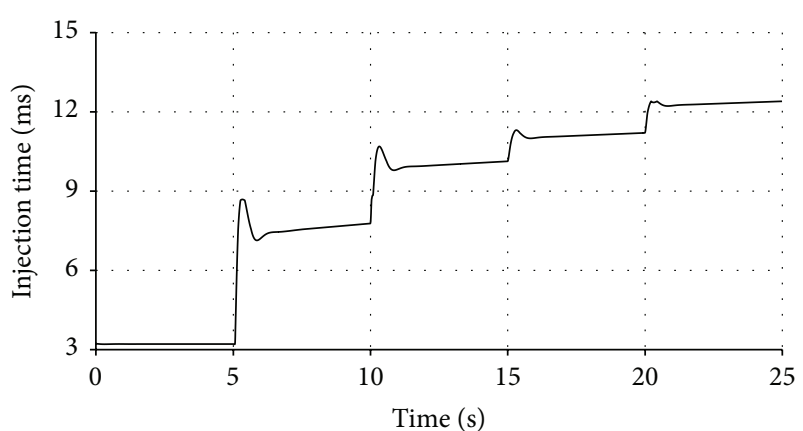

(b)

FIGURE 10: (a) Air-ratio control results and (b) corresponding fuel injection time of FTC under normal lambda sensor condition.

\section{Conclusions}

In this study, a supplementary air-ratio model was constructed using kernel-based ELM, in which the air-ratio time histories were not included in the model input. The purpose of the model is to predict the future air-ratio dynamics when the previous air-ratio measurement is unavailable. With the supplementary air-ratio model, a FTC for engine air-ratio control was developed as the first attempt in the literature. In traditional case, the lambda sensor is assumed to work properly, so traditional air-ratio control system may not perform well when the lambda sensor is malfunctioning. The purpose of the proposed FTC is to maintain the air-ratio control performance even when the lambda sensor is under failure or during cold start.

To evaluate its performance, the proposed FTC was successfully implemented and tested on a real automotive engine. Experimental results show that when the lambda sensor does not work well, the air-ratio control performance of the FTC can be effectively maintained over $90 \%$ of that under normal lambda sensor condition. Therefore, the FTC is a promising backup engine air-ratio control strategy. In other words, the proposed control scheme in Figure 3 is very suitable to implement in the automotive ECU to provide both regular and fault tolerance control of engine air-ratio.

\section{Conflict of Interests}

The authors declare that there is no conflict of interests regarding the publication of this paper.

\section{Acknowledgments}

The research is supported by the University of Macau Research Grant (Grant nos. MYRG2014-00178-FST and MYRG075(Y1-L2)-FST13-VCM). The research is also supported by the Science and Technology Development Fund of Macau (Grant no. FDCT/075/2013/A).

\section{References}

[1] J. Heinrich, P. E. Schwarze, N. Stilianakis et al., Health Effects of Transport-Related Air Pollution, World Health Organization, 2005.

[2] S. S. Lim, T. Vos, A. D. Flaxman et al., "A comparative risk assessment of burden of disease and injury attributable to 67 risk factors and risk factor clusters in 21 regions, 1990-2010: a systematic analysis for the Global Burden of Disease Study 2010," The Lancet, vol. 380, no. 9859, pp. 2224-2260, 2010.

[3] S. H. L. Yim and S. R. H. Barrett, "Public health impacts of combustion emissions in the United Kingdom," Environmental Science \& Technology, vol. 46, no. 8, pp. 4291-4296, 2012.

[4] K. Zhang and S. Batterman, "Air pollution and health risks due to vehicle traffic," Science of the Total Environment, vol. 450-451, pp. 307-316, 2013.

[5] J. B. Heywood, Internal Combustion Engine Fundamentals, McGraw-Hill, New York, NY, USA, 1988.

[6] T. Gilles, Automotive Service: Inspection, Maintenance, Repair, Cengage Learning, 2011.

[7] S. Pace and G. G. Zhu, "Sliding mode control of both air-tofuel and fuel ratios for a dual-fuel internal combustion engine," Journal of Dynamic Systems, Measurement and Control, vol. 134, no. 3, Article ID 031012, 2012.

[8] R. Tafreshi, B. Ebrahimi, J. Mohammadpour, M. A. Franchek, K. Grigoriadis, and H. Masudi, "Linear dynamic parametervarying sliding manifold for air-fuel ratio control in lean-burn engines," IET Control Theory \& Applications, vol. 7, no. 10, pp. 1319-1329, 2013.

[9] B. Ebrahimi, R. Tafreshi, H. Masudi, M. Franchek, J. Mohammadpour, and K. Grigoriadis, "A parameter-varying filtered PID strategy for air-fuel ratio control of spark ignition engines," Control Engineering Practice, vol. 20, no. 8, pp. 805-815, 2012.

[10] Y.-J. Zhai, D.-W. Yu, H.-Y. Guo, and D. L. Yu, "Robust air/fuel ratio control with adaptive DRNN model and AD tuning," Engineering Applications of Artificial Intelligence, vol. 23, no. 2, pp. 283-289, 2010.

[11] T. Sardarmehni, J. Keighobadi, M. B. Menhaj, and H. Rahmani, "Robust predictive control of lambda in internal combustion engines using neural networks," Archives of Civil and Mechanical Engineering, vol. 13, no. 4, pp. 432-443, 2013. 
[12] P. K. Wong, H. C. Wong, C. M. Vong, Z. Xie, and S. Huang, "Model predictive engine air-ratio control using online sequential extreme learning machine," Neural Computing and Applications, 2014.

[13] P. K. Wong, L. M. Tam, K. Li, and C. M. Vong, "Engine idlespeed system modelling and control optimization using artificial intelligence," Proceedings of the Institution of Mechanical Engineers, Part D: Journal of Automobile Engineering, vol. 224, no. 1, pp. 55-72, 2010.

[14] Y.-W. Kim, G. Rizzoni, and V. I. Utkin, "Developing a fault tolerant power-train control system by integrating design of control and diagnostics," International Journal of Robust and Nonlinear Control, vol. 11, no. 11, pp. 1095-1114, 2001.

[15] E. Gassenfeit and J. Powell, "Algorithms for air-fuel ratio estimation using internal combustion engine cylinder pressure," SAE Technical Paper 890300, 1989.

[16] J. R. Asik, G. M. Meyer, and D. X. Tang, "A/F ratio estimation and control based on induced engine roughness," IEEE Control Systems Magazine, vol. 16, no. 6, pp. 35-42, 1996.

[17] G.-B. Huang, "An insight into extreme learning machines: random neurons, random features and kernels," Cognitive Computation, vol. 6, no. 3, pp. 376-390, 2014.

[18] K. I. Wong, P. K. Wong, C. S. Cheung, and C. M. Vong, "Modelling of diesel engine performance using advanced machine learning methods under scarce and exponential data set," Applied Soft Computing, vol. 13, no. 11, pp. 4428-4441, 2013.

[19] J. W. Cao, Z. P. Lin, G.-B. Huang, and N. Liu, "Voting based extreme learning machine," Information Sciences, vol. 185, pp. 66-77, 2012.

[20] I. Lou, Z. Xie, W. K. Ung, and K. M. Mok, "Freshwater algal bloom prediction by extreme learning machine in Macau Storage Reservoirs," Neural Computing and Applications, 2014.

[21] J. Cao and L. Xiong, "Protein sequence classification with improved extreme learning machine algorithms," BioMed Research International, vol. 2014, Article ID 103054, 12 pages, 2014.

[22] P. K. Wong, K. I. Wong, C. M. Vong, and C. S. Cheung, "Modeling and optimization of biodiesel engine performance using kernel-based extreme learning machine and cuckoo search," Renewable Energy, vol. 74, pp. 640-647, 2015.

[23] T. R. Chandrupatla, "An efficient quadratic fit-sectioning algorithm for minimization without derivatives," Computer Methods in Applied Mechanics and Engineering, vol. 152, no. 12, pp. 211-217, 1998.

[24] W. W. Pulkrabek, Engineering Fundamentals of the Internal Combustion Engine, Pearson Prentice Hall, 2nd edition, 2004.

[25] D. Q. Mayne, J. B. Rawlings, C. V. Rao, and P. O. Scokaert, "Constrained model predictive control: stability and optimality," Automatica, vol. 36, no. 6, pp. 789-814, 2000.

[26] G. Y. Li, Application of Intelligent Control and MATLAB to Electronically Controlled Engines, Publishing House of Electronics Industry, Beijing, China, 2007. 


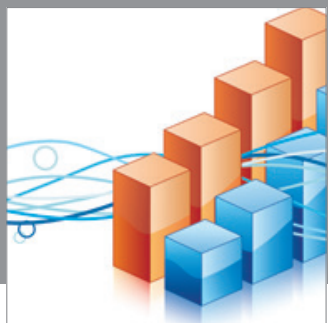

Advances in

Operations Research

mansans

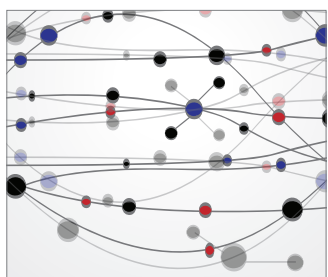

The Scientific World Journal
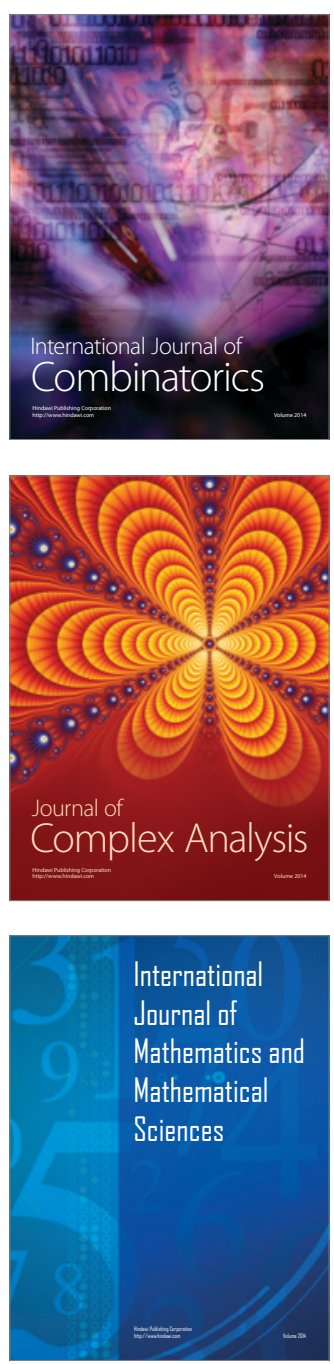
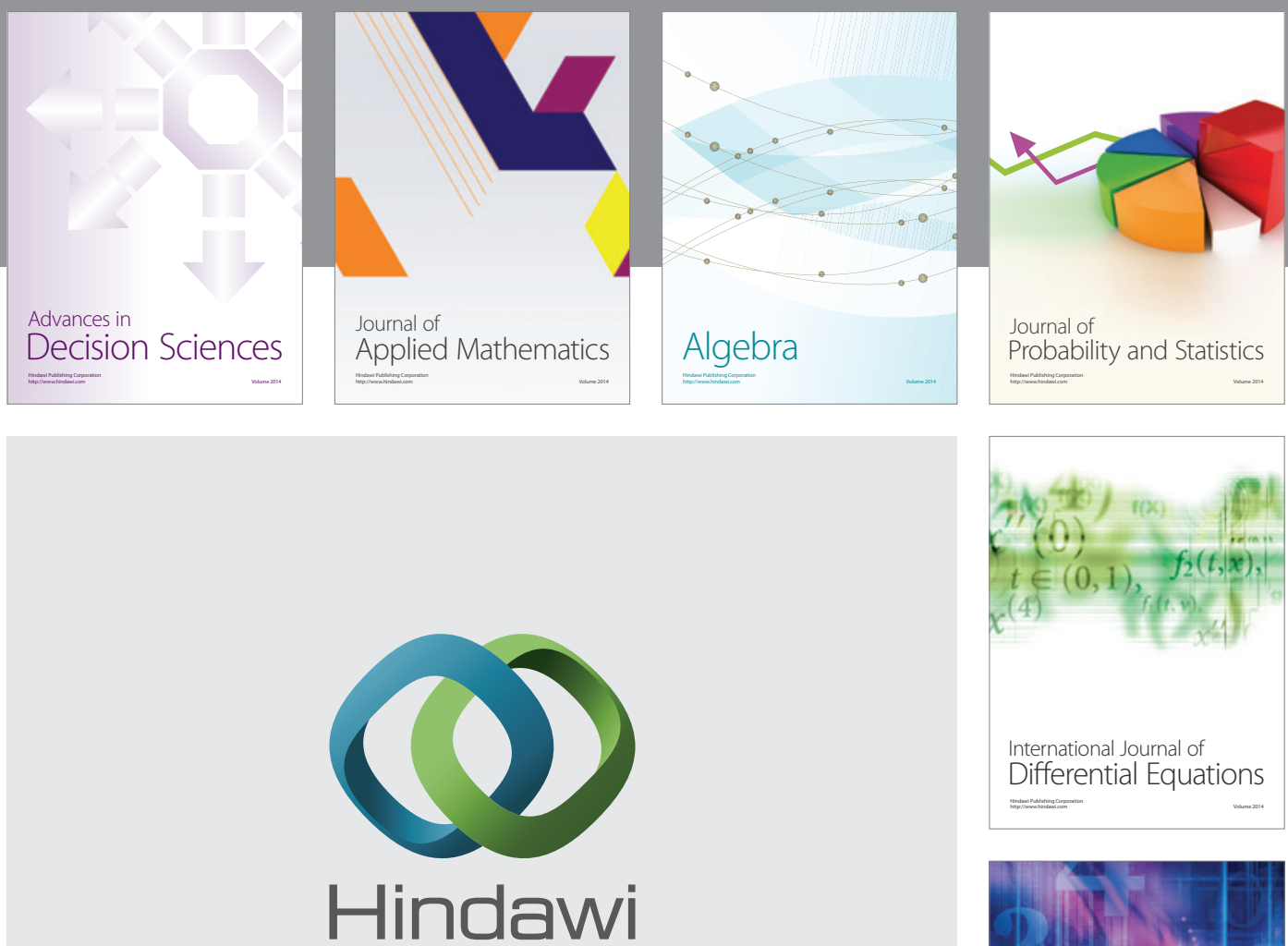

Submit your manuscripts at http://www.hindawi.com
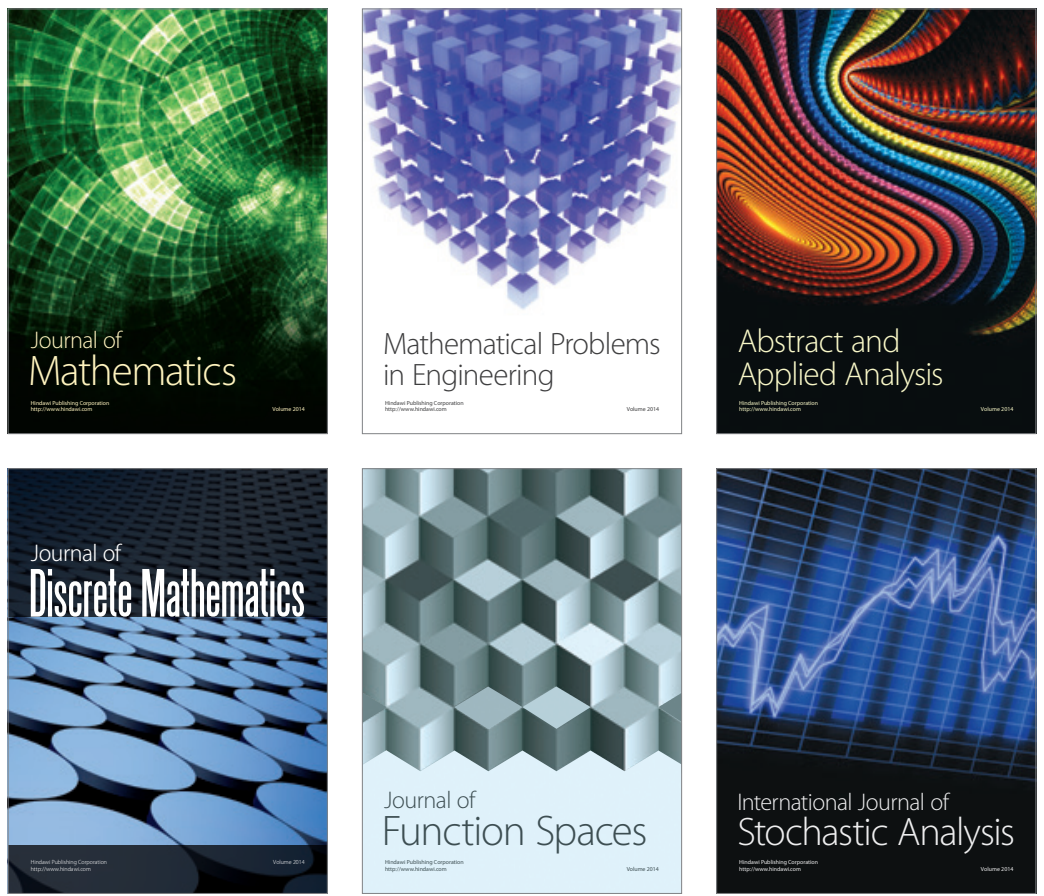

Journal of

Function Spaces

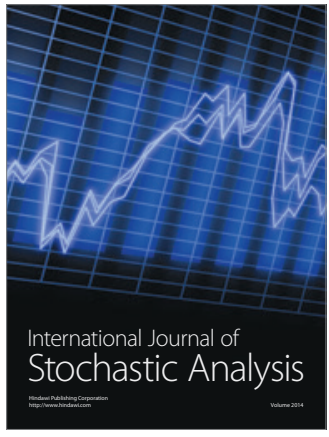

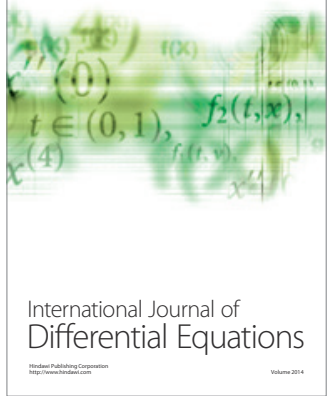
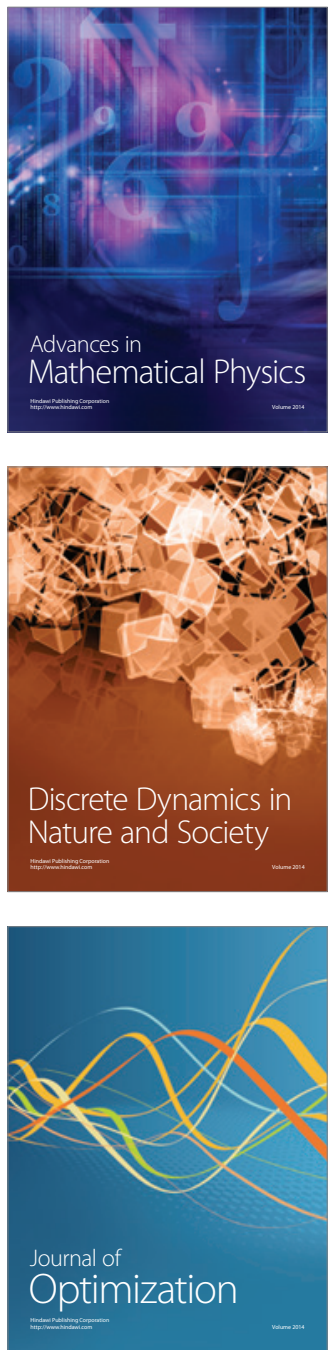